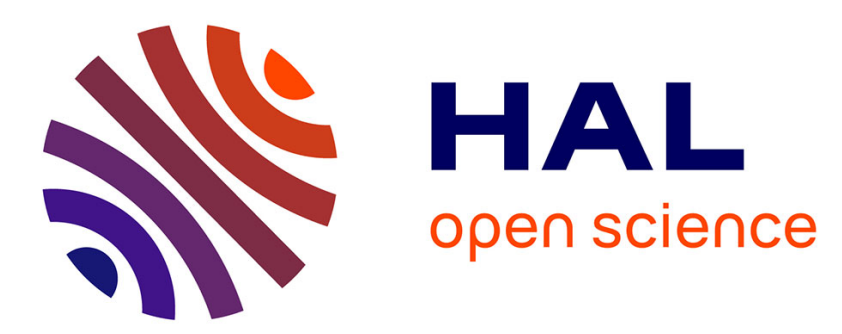

\title{
An equation of state for granular media at the limit state of isotropic compression
}

\author{
W. F Oquendo, J. Munoz, Farhang Radjai
}

\section{To cite this version:}

W. F Oquendo, J. Munoz, Farhang Radjai. An equation of state for granular media at the limit state of isotropic compression. EPL - Europhysics Letters, 2016, 114 (1), pp.14004. 10.1209/02955075/114/14004 . hal-02094934

\section{HAL Id: hal-02094934 https://hal.science/hal-02094934}

Submitted on 10 Apr 2019

HAL is a multi-disciplinary open access archive for the deposit and dissemination of scientific research documents, whether they are published or not. The documents may come from teaching and research institutions in France or abroad, or from public or private research centers.
L'archive ouverte pluridisciplinaire HAL, est destinée au dépôt et à la diffusion de documents scientifiques de niveau recherche, publiés ou non, émanant des établissements d'enseignement et de recherche français ou étrangers, des laboratoires publics ou privés. 


\title{
An equation of state for granular media at the limit state of isotropic compression
}

\author{
W. F. Oquendo ${ }^{1,2}$, J. D. Muñoz ${ }^{2}$ and F. RADJAI ${ }^{3,4}$ \\ ${ }^{1}$ Department of Industrial Engineering, Faculty of Engineering, Universidad de la Sabana \\ Km 7 Autopista Norte de Bogota, Chía, Colombia \\ 2 Simulation of Physical Systems Group, CEiBA-Complejidad, Department of Physics, \\ Universidad Nacional de Colombia - Carrera 30 No. 45-03, Ed. 404, Of. 348, Bogota D.C., Colombia \\ 3 LMGC, UMR5508, CNRS-University of Montpellier - 163 rue Auguste Broussonnet, 34090 Montpellier, France \\ ${ }^{4}\langle M S E\rangle^{2}$, MITei, Massachusetts Institute of Technology - 77 Massachusetts Avenue, Cambridge, MA 02139, USA
}

\begin{abstract}
It is well believed that the volumetric entropy of Edwards captures part of the physics of granular media, but it is still unclear whether it can be applied to granular systems under mechanical stress. By working out a recent proposal by Aste, Di Matteo et al. to measure Edwards' compactivity from the volume distribution of Voronoï or Delaunay tessellations (Phys. Rev. E, $7 \mathbf{7}$ (2008) 021309), and assuming that the total volume divides into elementary cells of fixed minimal volume, we derive an equation of state relating the compactivity to the packing fraction, and we show by extensive molecular-dynamics simulations that this equation and its underlying assumption describe well the volumetric aspects of both the limit state of isotropic compression and the limit state of shear (also called critical state in soil mechanics) for three-dimensional ensembles of monodisperse spheres, for a broad range of the sliding and rolling friction coefficients. In addition, by using the limit state of isotropic compression as testing ground, we find that the compactivity, the entropy per elementary cell and the number of elementary cells per grain computed by this method are the same within statistical precision, either by using Voronoï, Delaunay, or centroidal Voronoï tessellations, allowing thus for an objective definition. This means that not only Aste's cell method is robust and suitable to measure Edwards' compactivity of granular systems under mechanical stress but also the actual nature of the elementary cells might be unimportant.
\end{abstract}

Describing granular media within statistical mechanics is a long cherished dream [1]. Indeed, a disordered material composed of a large number of individual solid grains where we are just interested in their collective behavior seems to be a perfect candidate for a statistical description. This would be specially suitable for macroscopic states prepared by a well-defined protocol (like pluviation) or for limit states where the memory of the initial conditions has been lost, such as the limit state of isotropic compression or the limit state of simple shear. However, granular media are dissipative systems and the usual methods of equilibrium statistical mechanics do not apply directly.

One enlightening approach is Edwards' volume ensemble $[2-5]$. A granular material has the unique property of jamming in various states with different volumes (or packing fractions). If the volume is not restrained, it can change during a shear deformation (dilatancy). Edwards suggested that, in analogy to classical statistical systems, a microcanonical granular ensemble may be characterized by all jammed mechanically stable states of equal volume $V_{T}$ (instead of energy). Hence, a statistical mechanical approach may be initiated by assuming that all configurations of the same volume are equiprobable. The compactivity $\chi$ is the derivative of the volume with respect to entropy and, in this sense, replaces thermodynamical temperature.

Nevertheless, applying this theory is a challenging issue in itself because accounting for mechanical stability and measuring compactivity are not easy tasks. Furthermore, 
there is some evidence suggesting that the volume ensemble must be complemented by a description of forces to properly model granular media [6,7]. Nevertheless, in a recent work, Aste, Di Matteo and coworkers [4,5] introduced a promising method to compute the compactivity with good statistics from the volume distribution of either Voronoï or Delaunay cells. The method, to which we will refer below as "cell method", is based on the hypothesis that the total volume divides into elementary cells of volumes $v_{i}$, which characterize the volume distribution of the system, all elementary cells having a volume larger than or equal to a fixed minimal value $v_{\text {min }}$, due to the steric exclusions between particles and the volumes of the particles themselves. This method has been used to describe granular states created by pluviation [4] and to characterize the transition to crystallization [8], but it is not clear if this approach can be applied on samples under mechanical stress, like compression or shear, because it does not account for the forces. Here we show that the volumetric aspects of some reference states, like the limit states obtained by isotropic compression or by long time shearing, can be properly described by this theory. Moreover, we analytically derive an equipartition expression relating the volume fraction to the compactivity, and we show that it holds for the limit state of isotropic compression without tuning any adjusting parameter. In addition, the volumetric aspects of the limit state of shear (also known as critical state in soil mechanics) seem also to be described by the theory, but with a different value for the minimal volume $v_{\min }$ of an elementary cell.

Consider a sample of $N$ grains in a box of total volume $V_{T}$, in mechanical equilibrium with some external stress. Let us assume that $V_{T}$ is divided into $C$ elementary cells ${ }^{1}$ of similar shape and volume $v_{i}(i=1,2, \cdots, C)$, with $v_{i} \geq v_{\min }$ and $\sum_{i=1}^{C} v_{i}=V_{T}$, and that these volumes are the only independent degrees of freedom. Next, consider the configurational space where each free volume $v_{i}^{\prime} \equiv v_{i}-$ $v_{\text {min }}$ is a coordinate. We further assume that all ways to divide the total excess volume $V_{T}^{\prime} \equiv V_{T}-C v_{\text {min }}$ into the $C$ elementary cells are equally probable. The locus of all volume distributions with a total excess volume less than or equal to $V_{T}^{\prime}$ is a standard simplex of length $V_{T}^{\prime}$ and volume $\Sigma\left(V_{T}, C\right)=\left(V_{T}^{\prime}\right)^{C} /\left(\Lambda^{3 C} C\right.$ !), where we have introduced a reference length $\Lambda$ to make $\Sigma$ dimensionless. Therefore, the density of states $\Omega=\partial \Sigma / \partial V_{T}$ with total volume between $V_{T}$ and $V_{T}+\mathrm{d} V_{T}$ is

$$
\Omega\left(V_{T}, C\right)=\frac{\left(V_{T}-C v_{\min }\right)^{C-1}}{\Lambda^{3 C}(C-1) !} .
$$

The probability to find a specific elementary cell with a volume between $v$ and $v+\mathrm{d} v$ equals the fraction of all

\footnotetext{
${ }^{1}$ In two dimensions, these elementary cells might be identified with the quadrons proposed by Blumenfeld et al. [9,10], but this hypothesis needs to be tested.
}

configurations with this condition,

$$
\begin{aligned}
p(v) \mathrm{d} v & =\frac{\Omega\left(V_{T}-v, C-1\right)}{\Omega\left(V_{T}, C\right)} \frac{\mathrm{d} v}{\Lambda^{3 C}} \\
& =\frac{(C-1) / C}{\left(\langle v\rangle-v_{\min }\right)}\left(1-\frac{v-v_{\min }}{C\left(\langle v\rangle-v_{\min }\right)}\right)^{C-2} \mathrm{~d} v
\end{aligned}
$$

where $\langle v\rangle=V_{T} / C$. In the limit of $C \rightarrow \infty$, with $\langle v\rangle$ finite, this leads to

$$
p(v) d v=\frac{1}{\chi} e^{-\left(v-v_{\min }\right) / \chi} \mathrm{d} v
$$

where $\chi=\langle v\rangle-v_{\min }$ (the free volume per elementary cell) coincides with Edwards' compactivity [4,5].

Equation (3) can be used as in classical statistical mechanics to extract useful relations. For instance, given $p(v)=\exp (-v / \chi) / Z(\chi, 1)$ one can identify the partition function for a single elementary cell, $Z(\chi, 1)=$ $\frac{\chi}{\Lambda^{3}} \exp \left(-v_{\min } / \chi,\right)$, and the Gibbs entropy of a single elementary cell becomes

$$
\begin{aligned}
S(\chi, 1) & =-\int_{v_{\min }}^{\infty} p(v) \ln \left[\Lambda^{3} p(v)\right] \mathrm{d} v \\
& =\frac{\langle v\rangle}{\chi}+\ln Z(\chi, 1)=1+\ln \frac{\chi}{\Lambda^{3}} .
\end{aligned}
$$

If we assume, as a first approximation, that the $C$ cells are statistically independent, the volumetric entropy for the whole system will be $S(\chi, C)=C\left[1+\ln \left(\frac{\chi}{\Lambda^{3}}\right)\right]$, which fulfills $\partial S(\chi, C) / \partial V_{T}=1 / \chi$, as expected, because $\chi=$ $V_{T} / C-v_{\min }$. Actually, this last expression is an equipartition relation for the system.

Because we do not know exactly what the elementary cells are, it is not possible to measure $\chi$ directly. Instead, Aste, Di Matteo and coworkers proposed to divide the total volume into Voronoï, Delaunay or similar cells and to assume that each tessellation cell is composed of $k$ elementary cells [4]. Since the volume of each elementary cell has en exponential distribution (3), and they are assumed to be statistically independent, the volume $V$ of a tessellation cell will be distributed as a gamma distribution of parameter $k[4,5]$ :

$$
P_{\chi}(V)=\frac{k^{k}}{\Gamma(k)} \frac{\left(V-V_{\min }\right)^{k-1}}{\left(\langle V\rangle-V_{\min }\right)^{k}} e^{-k \frac{V-V_{\min }}{\langle V\rangle-V_{\min }}},
$$

where $V_{\min }=k v_{\min }$ is the minimal volume for a tessellation cell. Then, one can use the expected value $\langle V\rangle$ and the variance $\sigma_{V}^{2}$ of this distribution to estimate

$$
k=\frac{\left(\langle V\rangle-V_{\min }\right)^{2}}{\sigma_{V}^{2}}, \quad \chi=\frac{\sigma_{V}^{2}}{\langle V\rangle-V_{\min }},
$$

and $C=V_{T} /\left(\chi+V_{\min } / k\right)$. This shows that the whole distribution is uniquely determined by its first and second moments. The parameter $V_{\min }$ is known for Voronoï cells in monodisperse grains, but it should be fit for other cases, including polydisperse systems. 
From (6), one also obtains that the Gibbs entropy for a tessellation cell $[4,5]$,

$$
\left.S_{\text {cell }}=k 1+\ln \left(\frac{\langle V\rangle-V_{\min }}{k \Lambda^{3}}\right)\right],
$$

is just $k$ times the entropy of a single elementary cell (since the $k$ elementary cells are assumed statistically independent).

Other methods to compute $\chi$, like the volume fluctuations method $[11,12]$ or the overlapping histogram technique [13], can be analytically derived from the cell method. The volume fluctuations method computes the fluctuations of the total volume $\sigma_{V}^{2}$ as a function of $\langle V\rangle$ and integrates them to estimate differences in compactivity. Indeed, if $k$ is approximately constant (a good approximation as we will show later), then

$$
-\int_{V_{1}}^{V_{2}} \frac{\mathrm{d}\langle V\rangle}{\sigma_{V}^{2}[\langle V\rangle]}=-\int_{V_{1}}^{V_{2}} \frac{k \mathrm{~d}\langle V\rangle}{\left(\langle V\rangle-V_{\min }\right)^{2}}=\frac{1}{\chi_{2}}-\frac{1}{\chi_{1}},
$$

which is exactly the expression proposed by the volume fluctuations method $[11,12]$. Similarly, the overlapping histogram technique is based on the assumption that, at fixed compactivity, tessellation cell volumes distribute exponentially, a result that can also be derived from the cell method. Because the $k$ elementary cells in a tessellation cell are assumed statistically independent, the partition function for such a tessellation cell is just $Z(\chi, k)=$ $Z(\chi, 1)^{k}=\left(\frac{\chi}{\Lambda^{3}}\right)^{k} \exp \left(-V_{\min } / \chi\right)$. Thus, eq. (6) can be rewritten to show that the basic expression for the overlapping histogram method,

$$
\frac{P_{\chi_{1}}(V)}{P_{\chi_{2}}(V)}=\frac{Z\left(\chi_{2}, k\right)}{Z\left(\chi_{1}, k\right)} e^{-V\left(\frac{1}{\chi_{1}}-\frac{1}{\chi_{2}}\right)},
$$

holds $^{2}$. The ratio between volume distributions can be fitted to estimate compactivity differences, when both distributions overlap on a broad volume interval.

It is worth mentioning that in the case of Voronoï cells this "cell method" leads to simple and useful expressions. First, the minimal volume for a 3D Voronoï cell can be computed exactly as (see [14]) $V_{\min }^{\text {Voro }}=$ $5^{5 / 4} / \sqrt{2(29+13 \sqrt{5})} d^{3}$. Because $v_{\min }$ is also constant, it means that $k^{\text {Voro }}=C / N=V_{\min }^{\text {Voro }} / v_{\min }$ must be also a constant. Thus, one can multiply $\chi=\langle v\rangle-v_{\min }$ by $C /\left(N v_{\text {grain }}\right)$ on both sides (with $v_{\text {grain }}=\pi d^{3} / 6$ the volume of a single grain, $d$, the grain diameter) to obtain

$$
\frac{C}{N v_{\text {grain }}} \chi=\frac{1}{\nu}-\frac{V_{\text {min }}^{\text {Voro }}}{v_{\text {grain }}},
$$

where $\nu$ is the packing fraction (ratio of solid volume to total volume) and $V_{\min }^{\text {Voro }} / v_{\text {grain }} \simeq 1.3250$ is a constant.

\footnotetext{
${ }^{2}$ In ref. [13] it was stated that the $k$-gamma distribution was not able to predict the correct dependence between the compactivity and the mean volume. But that conclusion was based on an incorrect identification of the entropy as $S(V)=(k-1) \ln \left(V-V_{\min }\right)$.
}

Table 1: Setup for isotropic compression and simple shear under confining stress $\sigma_{\text {wall }}$.

\begin{tabular}{lc}
\hline Name & Value \\
\hline Number of particles $(N)$ & $10^{4}$ \\
Dimensionless stiffness $(\kappa)$ & $2 \times 10^{4}$ \\
Normal spring constant $\left(K_{n}\right)$ & $\kappa \sigma_{\text {wall }}$ \\
Normal restitution $\left(e_{n}\right)$ & 0.2 \\
Tangential spring constant $\left(K_{t}\right)$ & $0.8 K_{n}$ \\
Tangential restitution $\left(e_{t}\right)$ & 0.1 \\
Sliding friction $\left(\mu_{s}\right)$ & 0.3 \\
Rolling friction $\left(\mu_{r}\right)$ & 0.05 \\
\hline
\end{tabular}

Even more, by comparing this expression with that for the $\mathrm{RCP}$ (and taking $\nu_{\mathrm{RCP}}=0.64$, see $[15,16]$ ), we arrive at

$$
\chi=A\left(\frac{1}{\nu}-\frac{V_{\text {min }}^{\text {Voro }}}{v_{\text {grain }}}\right),
$$

with $A=\chi_{\mathrm{RCP}} /\left(\frac{1}{\nu_{\mathrm{RCP}}}-\frac{V_{\mathrm{min}}^{\text {Voro }}}{v_{\text {grain }}}\right) \simeq 0.04481 d^{3}$. This is a state equation between the compactivity and the packing fraction. Interestingly, this equation involves no free parameter to be adjusted. This shows a possible minimum parameter set necessary to describe the system state $[7,17,18]$.

In view of applying the above theory to granular systems under mechanical stress, we performed extensive threedimensional simulations on a packing of mono-disperse spheres in two limit states: the limit state of isotropic compression and the limit state of shear, with several values of the coefficients of rolling $\mu_{r}$ and sliding $\mu_{s}$ friction. The rolling friction is implemented as in [19], where you should multiply by the radius to get a maximum torque. All numerical samples were prepared from a dilute and randomly disordered cubic sample (packing fraction $\simeq 0.20$ ). To ensure reproducibility (i.e. independence from the initial conditions) and homogeneity both in stress and fabric for the final state $[20,21]$, we applied a quasi-static compression with very low inertial number $I=\dot{\epsilon} d \sqrt{\rho_{m} / P} \simeq$ $5 \times 10^{-4}$, where $\dot{\epsilon}$ is the deformation rate, $d$ the mean diameter, $\rho_{m}$ the mass density, and $P$ the external pressure. Moreover, special care was taken to assure that crystallization was absent in the sample $[22,23]$. This limit state of isotropic compression was also the initial state for the shear, which was also driven with $I \simeq 5 \times 10^{-4}$ until both the volume fraction and shear stress level off. We used the soft-particle discrete-element method [21] with a high dimensionless stiffness $\kappa=K_{n} / d \sigma_{\text {wall }}=2 \times 10^{4}$, where $K_{n}$ is the grain elastic constant and $\sigma_{\text {wall }}$ is the final value of the pressure applied on the six faces of the cubic sample (for isotropic compression) or on the top-bottom walls (for the shear) [20,21]. All other simulation parameters are listed in table 1 . Once the total volume reaches its final value, the spatial tessellations are constructed for the inner particles (three diameters away from the boundaries) and the statistical variables are computed. Each simulation 


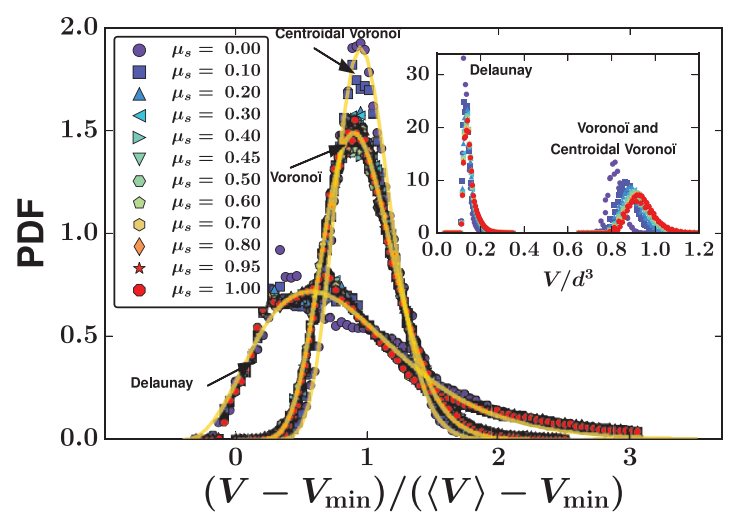

Fig. 1: (Color online) Volume distributions of Voronoï, centroidal Voronoï and Delaunay cells in the limit state of isotropic compression, with $\mu_{r}=0.05$ as example, and several values of $\mu_{s}$. Results are similar for other values of $\mu_{r}$. The inset shows the original volume distributions without normalization. The continuous lines represent eq. (6) with $k=12, k=22$, and $k=2.0$ for Voronoï, centroidal Voronoï, and Delaunay cells, respectively. The average chi-square agreement between the continuous lines and the measured distributions is 0.029(1).

was repeated eight times with different initial grain positions to compute error bars. This protocol resembles typical laboratory procedures, where the material is compressed from an initial looser state compared to methods like the Lubachevsky-Stillinger or Jodrey-Tory protocols where particles are grown in size at a given speed until some specified density is achieved [24,25].

The first main result we obtained is that Voronoï, Delaunay and centroidal Voronoï cells do distribute as $k$-gamma (fig. 1), with a different value of $k$ for each cell type; that is, the elementary cell theory actually applies to frictional granular systems under mechanical stress. Results are similar for other values of $\mu_{r}$ (see footnote ${ }^{3}$ ).

Regarding the entropy, and since only differences in entropy are physically meaningful, one can choose a reference state, for instance the random close packing (RCP) [18,26], and define $\Delta S=S-S_{\mathrm{RCP}}=\ln \chi-\ln X_{\mathrm{RCP}}$. Choosing the RCP as reference state with null entropy, since it corresponds to a maximum density limit, see [27], leads to $\Lambda=\sqrt[3]{\mathrm{e} \chi \mathrm{RCP}}$ in eq. (4), where $\mathrm{e}$ is the base of natural logarithms. Our measurements on a RCP, obtained through the isotropic compression of $\simeq 10000$ frictionless particles $^{4}$ (also shown in fig. 1) yield $\chi_{\mathrm{RCP}} / d^{3}=0.0101(1)$ by using Voronoï cells and $\chi_{\mathrm{RCP}} / d^{3}=0.0104(4)$ by using Delaunay ones. This value corresponds to $\Lambda / d \simeq 0.301$, giving support to the value used by [8].

\footnotetext{
${ }^{3}$ For the Voronoi cells all cells are included in the analysis; in contrast, $v_{\text {min }}^{\text {Dela }}$ is computed by fitting the $k$-gamma complementary cumulative distribution, and only cells with $V \geq V_{\text {min }}^{\text {Dela }}$ are included. This last procedure was validated by reproducing the values of $\chi$ gathered by Aste et al. [5] for an experimental sample of $\simeq 90000$ grains prepared by pluviation.

${ }^{4}$ The RCP was identified by verifying that the order metrics $Q_{4}$ and $Q_{6}$ took the characteristics of the maximally random jammed state reported by $[23,28]$.
}

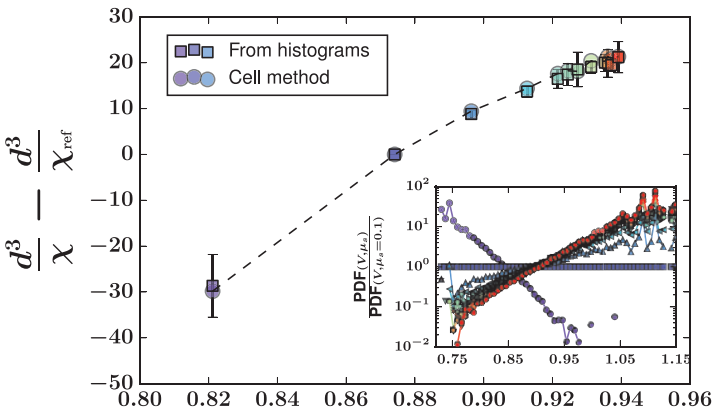

$\langle V\rangle / d^{3}$

Fig. 2: (Color online) Compactivity differences computed from both the cell method (circles, eq. (7)) and overlapping histograms method (squares, eq. (9)), for several values of $\mu_{s}$, constant $\mu_{r}=0.05$, and a reference value at $\mu_{s, \text { ref }}=0.1$. The inset shows, in semi-log scale, that the ratio between the distributions of total volumes at different mean volumes are approximately exponential within the overlapping range, as the overlapping histograms method assumes. The $\mu_{s}$ values are color-encoded as in fig. 1.

Computing compactivities and entropies is easier with the cell method, because it only needs the volume distribution, and coincides with the volume fluctuations and overlapping histograms methods. Figure 2 shows a good agreement among the compactivities obtained from the cell method and those obtained from the overlappinghistogram method. The results are computed for several values of the sliding friction coefficient, with a constant rolling friction coefficient $\mu_{r}=0.05$ and fixing $\chi$ for $\mu_{s}=0.1$ as reference value for the overlapping-histogram method. Similar results are obtained for different values of $\mu_{r}$ and for other reference values. The inset shows the histograms ratios in semi-log scale, which indicates an exponential trend in the overlapping region.

The next step is to establish that the predictions of the model are independent of tessellation. If this is true, it will provide a strong support of this method for the estimation of compactivities, which are not only independent of the tessellation but also coincide quantitatively with other approaches, as shown before. To show this property, we tessellated the total volume by three different methods: the Voronoï tessellation, the Delaunay tessellation, and the centroidal Voronoï tessellation [14,29]. Figure 3 shows the compactivity $\chi$, the entropy per elementary cell $S(\chi, 1)=S(\chi, k) / k$, and $C / N$, as functions of the sliding friction coefficient $\mu_{s}$, obtained with $\mu_{r}=0.05$ from the three tessellations. All of them give the same results within error bars. In addition, $C / N$ (which equals $k$ for Voronoï cells) shows small error bars and is almost constant for $\mu_{s} \geq 0.2$, as the cell method requires. These results show that these three statistical quantities are independent of the tessellation employed to measure them. The inset shows the entropy per tessellation cell, which actually differs because the number $k$ of elementary cells per tessellation cell is different for each one. 

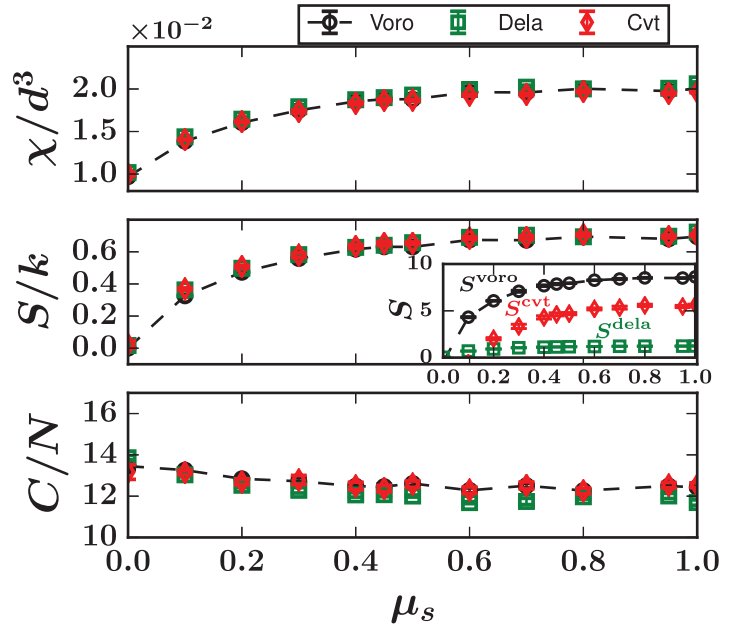

Fig. 3: (Color online) Compactivity $\chi$, entropy per elementary cell $S(\chi, 1)=S / k$, and number of elementary cells per grain $C / N$ at the limit state of isotropic compression, as functions of the sliding friction coefficient $\mu_{s}$, with fixed $\mu_{r}=0.05$, as computed from Voronoï (black circles), Delaunay (green squares), and centroidal Voronoï (red diamonds) tessellations. The inset shows the entropy $S$ which is different for each tessellation.

This independence with respect to the tessellation was confirmed on simulations with many more combinations of $\mu_{s}$ and $\mu_{r}$. Figure 4 displays $\chi$ as a function of $\mu_{s}$ and $\mu_{r}$, computed from the same three tessellations. The agreement is again excellent (within error bars). As expected, increasing the sliding or rolling friction coefficients leads to a looser state of higher compactivity [30,31], but the compactivity increases up to a saturation value, since the grains must keep a minimum number of contacts in order to sustain the external pressure ${ }^{5}$.

Finally, a major result of the present work is to establish an equation of state (actually an equipartition relation) between the compactivity $\chi$ and the packing fraction $\nu$, where no fit parameters are involved (eq. (12)). The larger the packing fraction, the smaller the compactivity. Figure 5 displays $\chi$ as a function of $1 / \nu$. We see that a rather large number of numerical data for the limit state of isotropic compression collapse over the trend stated by eq. (12), even for extreme values of the rolling friction up to $\mu_{r}=0.50$ (within error bars). The fact that so many systems of stiff grains with such different values of $\mu_{r}$ and $\mu_{s}$ fit all of them in the same equation of state suggests that the volumetric and force-network aspects of a granular systems in the limit of hard spheres might be studied separately.

The actual mechanical nature of the system is reflected on the prefactor $A$ of the state equation, since the reference state does not need to be the RCP if the limit state is not the one of isotropic compression. For example, fig. 5 also shows some data from bi-periodic simple

\footnotetext{
${ }^{5}$ In contrast, varying $\kappa$ from 200 to 40000 (at a fixed confining pressure $\sigma_{\text {wall }}=10^{4} \mathrm{~Pa}$ ) does not affect $\chi$, but it affects $C / N$, which increases and saturates for increasingly stiff spheres
}

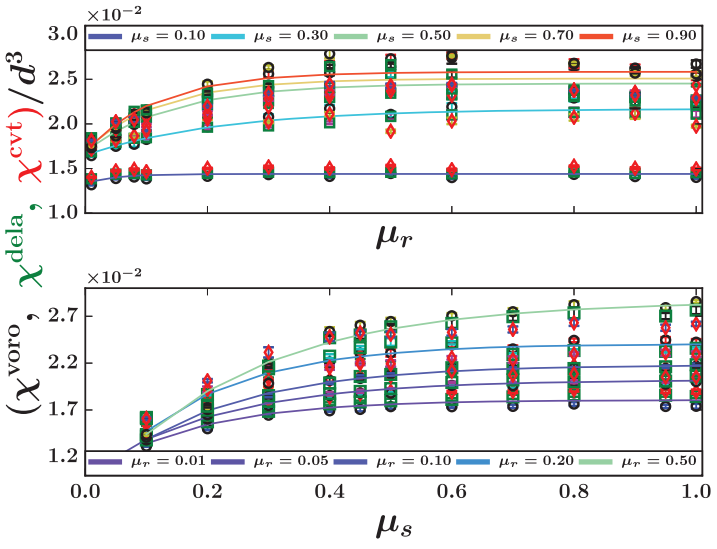

Fig. 4: (Color online) Compactivity $\chi / d^{3}$ as a function of rolling, $\mu_{r}$, and sliding, $\mu_{s}$, friction coefficients, computed from Voronoï (diamonds), Delaunay (squares), and centroidal Voronoï (circles) tessellations. As guide to the eyes, the solid lines are exponential functions.

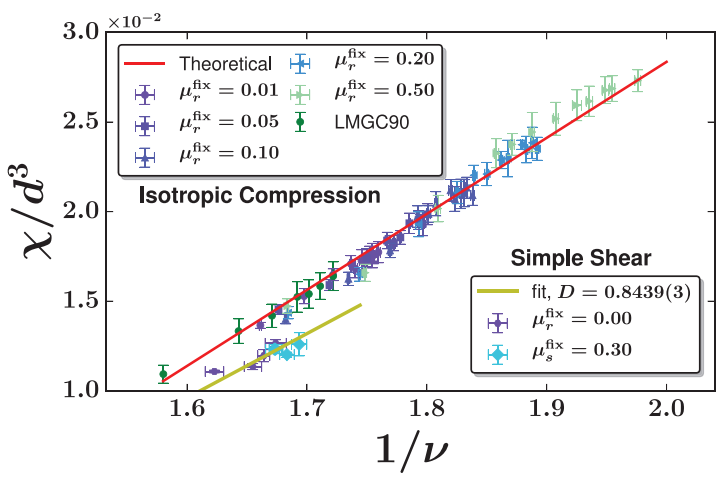

Fig. 5: (Color online) Compactivity $\chi / d^{3}$ as a function of the inverse of packing fraction $1 / \nu$, for both the limit states of isotropic compression and simple bi-periodic shear for monodisperse spheres. Each color represents simulations with a fixed value of $\mu_{r}$ and several values of $\mu_{s}$ between 0.1 and 1.0. The solid line is the prediction of eq. (12). The dashed line represents a fit of the theoretical prediction for simple shear tests. Some additional data obtained with the LMGC90 contact dynamics code [32] are also included.

shear simulations. These results are outside eq. (12), but, they follow a similar linear trend. This can be explained considering that the actual RCP state does not represent a sheared system in terms of internal system geometry and volume partition. A frictionless simple-sheared system $[33,34]$, which actually has a non-zero macroscopic friction, would do better. In this case, the actual value for $\chi_{\text {ref }}$ will be smaller, and, therefore, the equation of state will have a smaller slope, as observed in fig. 5 .

It is important to note that $k=C / N \simeq 12$ for Voronoï cells, which is also the average number of first neighbors for a monodisperse system in 3D; a clue for future works to establish what actually elementary cells are.

In summary, by extensive three-dimensional MD simulations of sphere packings on broad ranges of sliding and 
rolling coefficients, we showed, first, that the cell method does also describe samples under mechanical stress and, second, that the main statistical quantities describing a packing in this theory (compactivity, entropy per elementary cell and mean number of elementary cells per grain) are independent of the tessellation method. This finding provides a strong support for the objectivity of the cell method and Edwards' entropy. These quantities appear thus as suitable parameters for describing these limit states. Next, using this framework, we derived an analytic expression for the total volumetric entropy, and an equation of state relating the compactivity and the packing fraction in close agreement with numerical data. This equation provides a means to measure the compactivity from volume fractions. More importantly, it can be used in conjunction with constitutive modeling of quasi-static rheology of granular materials, which is often based on phenomenological approaches. In particular, our equation of state may be extended to include memory effects or, at least, it can be used to quantify such effects under complex loading conditions.

\section{$* * *$}

We would like to thank Dr. N. EstradA, at Universidad de Los Andes, Colombia, for very helpful discussions and Prof. T. Aste for giving us access to the experimental data to calibrate our results. JDM also thanks Prof. H. J. Herrmann for hospitality in Switzerland. This work was partially supported by the Excellence Center for Basic and Applied Research on Complexity, CEiBAComplejidad, the DIB-UNAL, and the Fundación para la Promoción de la Ciencia y la Tecnología del Banco de la República, Colombia. WFO thanks the LMGC for support during his stay in France, where part of this work was completed.

\section{REFERENCES}

[1] Cowin S. C. and Satake M., J. Rheol., 23 (1979) 243.

[2] Edwards S. F. and Oakeshott R. B. S., Physica A: Stat. Theor. Phys., 157 (1989) 1080.

[3] Hinrichsen H. and Wolf D. E. (Editors), The Physics of Granular Media (Vch Verlagsgesellschaft Mbh) 2004.

[4] Aste T. and DiMatteo T., Phys. Rev. E, 77 (2008) 021309.

[5] Aste T., Di Matteo T., Saadatfar M., Senden T. J., SChröter M. and Swinney H. L., EPL, 79 (2007) 24003.

[6] Blumenfeld R., Jordan J. F. and Edwards S. F., Phys. Rev. Lett., 109 (2012) 238001.

[7] Pugnaloni L. A., Sánchez I., Gago P. A., Damas J., Zuriguel I. and Maza D., Phys. Rev. E, 82 (2010) 050301.
[8] Anikeenko A. V., Medvedev N. N. and Aste T., Phys. Rev. E, 77 (2008) 031101.

[9] Hininashvili R. and Blumenfeld R., Granular Matter, 14 (2012) 277.

[10] Frenkel G., Blumenfeld R., Grof Z. and King P. R., Phys. Rev. E, 77 (2008) 041304.

[11] Nowak E. R., Knight J. B., Ben-Naim E., Jaeger H. M. and Nagel S. R., Phys. Rev. E, 57 (1998) 1971.

[12] Schroter M., Goldman D. I. and Swinney H. L., Phys. Rev. E, 71 (2005) 030301.

[13] McNamara S., Richard P., DE Richter S. K., Le Caër G. and Delannay R., Phys. Rev. E, 80 (2009) 031301.

[14] Aste T. and Weaire D., The Pursuit of Perfect Packing (Taylor \& Francis Group) 2008.

[15] Jin Y. and Makse H. A., Physica A: Stat. Mech. Appl., 389 (2010) 5362.

[16] Song C., Wang P. and Makse H., Nature, 453 (2008) 629.

[17] Fierro A., Nicodemi M. and Coniglio A., Phys. Rev. E, 66 (2002) 061301.

[18] Ciamarra M., Richard P., Schröter M. and Tighe B., Soft Matter, 8 (2012) 9731.

[19] Luding S., Granular Matter, 10 (2008) 235.

[20] Agnolin I. and Roux J.-N., Phys. Rev. E, 76 (2007) 061302.

[21] Radjaï F. and Dubois F. (Editors), Discrete-element Modeling of Granular Materials (Wiley) 2011.

[22] Panaitescu A., Reddy K. A. and Kudrolli A., Phys. Rev. Lett., 108 (2012) 108001.

[23] Kapfer S. C., Mickel W., Mecke K. and SchröderTurk G. E., Phys. Rev. E, 85 (2012) 030301.

[24] Stillinger F. H. and Lubachevsky B. D., J. Stat. Phys., 73 (1993) 497.

[25] Parisi G. and Zamponi F., Rev. Mod. Phys., 82 (2010) 789.

[26] Shahinpoor M., Powder Technol., 25 (1980) 163.

[27] Ciamarra M. P. and Coniglio A., Phys. Rev. Lett., 101 (2008) 128001.

[28] Torquato S., Truskett T. M. and Debenedetti P. G., Phys. Rev. Lett., 84 (2000) 2064.

[29] Du Q., Faber V. and Gunzburger M., SiaM Rev., 41 (1999) 637.

[30] Estrada N., Taboada A. and Radjai F., Phys. Rev. E, 78 (2008) 021301.

[31] Azéma E., Estrada N. and Radjai F., Phys. Rev. E, 86 (2012) 041301.

[32] Dubois F., Jean M., Renouf M., Mozul R., Martin A. and Bagneris M., Lmgc90, in Proceedings of the 10e colloque national en calcul des structures, 2011, https://hal.archives-ouvertes.fr/hal00596875/.

[33] Peyneau P.-E. and Roux J.-N., Phys. Rev. E, 78 (2008) 011307.

[34] AzÉma É., Radjaï F. and Roux J.-N., Phys. Rev. E, 91 (2015) 010202. 